\title{
Napping Characteristics and Cognitive Performance in Older Adults
}

\author{
Jocelynn T. Owusu, MPH ${ }^{1}$, Alexandra M. V. Wennberg, $\mathrm{PhD}^{2}$, Calliope B. Holingue, MPH ${ }^{1}$, \\ Marian Tzuang, MSW ${ }^{1}$, Kylie D. Abeson, MPH $^{1}$, and Adam P. Spira, Ph.D. ${ }^{1,3,4}$ \\ ${ }^{1}$ Department of Mental Health, Johns Hopkins Bloomberg School of Public Health \\ ${ }^{2}$ Department of Health Sciences Research, Mayo Clinic, Rochester, MN \\ ${ }^{3}$ Department of Psychiatry and Behavioral Sciences, Johns Hopkins School of Medicine \\ ${ }^{4}$ Center on Aging and Health, Johns Hopkins University
}

\section{Abstract}

Objectives: To determine the association of napping intention, frequency, and duration with cognition in a nationally-representative sample of U.S. older adults.

\begin{abstract}
Methods: We performed a cross-sectional analysis of community-dwelling Medicare beneficiaries aged $\ 65$ years from Rounds 3 or 4 (2013-2014) of the National Health and Aging Trends Study $(\mathrm{N}=2,549)$. Participants reported past-month napping intention (intentional/ unintentional), napping frequency (rarely/never [non-nappers], some days [infrequent nappers], most days/every day [frequent nappers]), and average nap duration (we categorized as $\mathbf{3 0}$ minutes [short]; 31-60 minutes [moderate]; and >60 minutes [long]). Cognitive outcomes were performance on immediate and delayed word recall tests (IWR and DWR, respectively), the Clock Drawing Test (CDT), and self-rated memory (score: 1[excellent]-5[very poor]).
\end{abstract}

Results: After adjustment for potential confounders, unintentional nappers had poorer immediate word recall test performance than non-nappers $(\mathrm{B}=-0.23, p<0.01)$ and intentional nappers $(\mathrm{B}=$ $-0.26, p<0.01)$. After further adjustment for daytime sleepiness, frequent nappers reported poorer self-rated memory than non-nappers $(\mathrm{B}=0.14, p<0.05)$. Compared to short nappers, long nappers had poorer IWR $(\mathrm{B}=-0.26, p<0.05)$ and $\mathrm{CDT}$ scores $(\mathrm{B}=-0.17, p<0.05)$. Except for the association of nap duration with IWR and CDT, these associations remained after excluding participants with dementia and/or proxy respondents. Among participants undiagnosed with dementia or proxies, moderate-duration naps were associated with better DWR than short naps $(\mathrm{B}=0.24, p<0.05)$. Neither napping intentionality nor frequency were associated with CDT performance.

Conclusions: Among older adults, distinct aspects of napping are associated with cognitive performance. Prospective research, with objective measures of napping, is needed to elucidate the link between napping and cognitive trajectories.

Corresponding Author: Jocelynn T. Owusu, MPH; Address: 624 North Broadway, Room 802, Baltimore, MD 21205, jowusu1@jhu.edu.

The other authors report no conflicts of interest. 


\section{Keywords}

nap; sleep; cognitive function; dementia

\section{Introduction}

The U.S. population of adults aged $\succeq 65$ years is projected to grow from 46 million in 2014 to 98 million by $2060 .{ }^{1}$ Given that age is the greatest risk factor for dementia ${ }^{2}$, there will be an increase in the population burden of cognitive impairment. Approximately $5-7 \%$ of adults aged $\succ 60$ years have dementia in most regions of the world, including the U.S. ${ }^{3}$ Therefore, there is a critical need to identify those at risk for cognitive decline and dementia to enhance care planning. Additionally, there are no treatments with a meaningful effect on the dementia course, necessitating the identification of modifiable risk factors that can be manipulated to prevent dementia onset or slow its progression.

A growing body of studies suggests an association of disturbed sleep with an increased risk of cognitive decline and dementia, ${ }^{4}$ but less attention has been paid to napping. Up to $49 \%$ of older adults report regular napping, and this increases with age. ${ }^{5-7}$ Interestingly, both positive and negative associations have emerged between napping and cognitive performance. For example, an observational study found that, compared to older adults who did not nap, those reporting any napping or napping $₹ 60$ minutes had a decreased risk of decline, as determined by Mini-Mental State Exam scores. ${ }^{8}$ In laboratory-based experiments, napping was associated with improved reaction time, ${ }^{9}$ processing speed, and logical reasoning among older adults. ${ }^{10}$ However, among older adults, studies using actigraphy have found links between longer naps and poorer cognitive performance, ${ }^{11,12}$ and reports of unintentional naps have been linked to poorer performance on tests of attention and concentration, temporal orientation, and delayed recall in unadjusted models. ${ }^{6}$

The mixed evidence linking napping and cognition may be partially attributable to the use of limited measures of napping, such as binary measures of napping (yes/no) or nap duration. $8,11,13,14$ Napping, however, can vary by frequency, duration, timing and intentionality, which these coarser measures do not capture. Napping intention is important to study because intentional naps are qualitatively different from unintentional naps. The latter are presumably due to excessive daytime sleepiness (EDS), ${ }^{15}$ which can be due to insufficient or otherwise disturbed sleep. On the other hand, intentional naps may simply be taken for pleasure. Further research is needed to determine whether distinct dimensions of napping influence, or are an indicator of, health among older adults. Indeed, napping may be a modifiable behavior that could be leveraged to improve cognitive outcomes among older adults, ${ }^{8}$ or to identify those at higher risk for cognitive decline. To begin to investigate this possibility, we studied the cross-sectional association of napping frequency, intention, and duration with cognitive outcomes in a nationally representative sample of communitydwelling older adults in the U.S. 


\section{Methods}

\section{Participants}

Participants were enrolled in the National Health and Aging Study (NHATS), a representative sample of U.S. Medicare beneficiaries aged $\succeq 5$ years. ${ }^{16}$ NHATS protocols were reviewed and approved by the Johns Hopkins Bloomberg School of Public Health Institutional Review Board. At the time of the NHATS visit, NHATS administered a sleepfocused module that included napping-related items to a random subset of participants from Rounds 3 and 4 (2013-2014). We combined data from these Rounds, yielding 2,908 participants with napping data. When participants were not able to respond to NHATS study questions due to illness, disability, or a language barrier, a proxy respondent answered study questions for them. However, when possible, participants with proxy respondents completed cognitive assessments. Our sample included 54 participants with a proxy respondent. We excluded 234 participants residing in long-term care (i.e., nursing homes, other residential care), 107 participants missing data for all cognitive measures, and 18 participants missing demographic data for a final sample of 2,549 participants.

\section{Sleep Characteristics}

Napping.-Participants responded to items concerning past-month napping. One item asked, "...how often did you take naps during the day?" (responses: "never", "rarely", "some days", "most days", "every day"). We coded participants who never or rarely napped as "non-nappers", those who napped some days as "infrequent" nappers, and those who napped most days or every day as "frequent" nappers. Participants who reported napping more than "never" were asked additional nap-related questions. The first was, "On average, how long were these naps?". We categorized participants by nap duration: $\mathfrak{3 0}$ minutes (short), 31-60 minutes (moderate), or >60 minutes (long). The second question was, "In general, were these naps planned, or did you fall asleep without meaning to?" Response options were: "naps planned," "fell asleep without meaning to," "both (some planned/some not)". We classified participants reporting "both" or "fell asleep without meaning to" as "unintentional" nappers, and those reporting planned naps only as "intentional" nappers. We did not analyze duration and intention data for participants who reported napping "rarely".

Daytime Sleepiness.—Participants were asked, "During the past month, how often did you have trouble staying awake at times during the day when you wanted to be awake?" (responses: "never", "rarely", "some days", "most days", or "every day").

Nighttime Sleep Duration.-Participants were asked, "During the past month, how many hours of actual sleep did you usually get at night?" Participants who were unable to provide usual nighttime sleep duration because they didn't have a usual sleep duration (their sleep duration varied from night to night) did not receive a numeric value for hours of usual sleep. Therefore, these participants $(n=53)$ were excluded from sleep duration analyses.

Use of Medication for Sleep.-Participants were asked, "During the past month, how often did you take medication to help you sleep?" (responses: "never", "rarely", "some nights", "most nights", "every night"). 


\section{Cognitive Assessments}

Word List Memory Test.-Participants completed the Word List Memory test during the NHATS interview. ${ }^{17}$ Trained interviewers read 10 words to participants and asked them to recall as many as possible immediately afterwards (immediate word recall (IWR)) and five minutes later (delayed word recall (DWR)). The number of words correctly recalled for each test served as the score (range 0 to 10 for each).

Clock Drawing Test.-Participants completed the Clock Drawing Test (CDT) during the NHATS interview. The CDT measures executive function and visuospatial ability. Participants were allotted two minutes to draw a clock with the hands pointing to " 10 after 11 ". Drawings were scored from 0 ("not recognizable as a clock") to 5 ("accurate depiction of a clock") using standardized criteria. ${ }^{18}$

Self-Rated Memory.-Participants were asked, "How would you rate your memory at the present time?" with response options: 1 (“excellent"), 2 ("very good"), 3 (“good"), 4 ("fair"), or 5 ("poor").

\section{Demographics and Health Characteristics}

Age, gender, race, and educational attainment were obtained on NHATS enrollment. NHATS classifies age in categories (65-69 years; $70-74$ years; $75-79$ years; $80-84$ years; 85-89 years; 90+ years), and race as White, non-Hispanic; Black, non-Hispanic; Hispanic; $>1$ race/ethnicity (no primary race was reported), and Other (e.g., American Indian, Native Hawaiian, Asian, Pacific Islander). We combined the "Other" and "> 1 race" category. We classified educational attainment as <high school, high school graduate, or >high school. We calculated body mass index (BMI; $\mathrm{kg} / \mathrm{m}^{2}$ ) using self-reported height and weight and categorized it as: <18.5 (underweight), 18.5-24.9 (normal weight), 25-29.9 (overweight), or $\geq 30$ (obese).${ }^{19} \mathrm{We}$ categorized participants by the number of the following medical conditions they reported being told they have by a healthcare provider ( 0 or 1, 2, 3, 4+): heart attack; heart disease; high blood pressure; arthritis; osteoporosis; diabetes; stroke; dementia or Alzheimer's disease (AD); and cancer. Depressive and anxiety symptoms were measured by the Patient Health Questionnaire (PHQ-2) and the Generalized Anxiety Disorder (GAD-2), respectively. ${ }^{20}$ These are both two-item measures; responses range from 0 to 3 per item. We summed responses to obtain a score (range 0 to 6 for each measure).

\section{Statistical Analyses}

We categorized participants by napping type: non-nappers, intentional, and unintentional nappers, and compared their characteristics using chi-square tests (categorical variables) and simple linear regression (continuous variables). IWR, DWR, and CDT scores, as well as self-rated memory, were analyzed as continuous variables. Higher scores indicated poorer self-rated memory. Covariates were added to our models based on a priori knowledge of their roles as potential confounders or on empirical evidence from our data (association with at least one predictor and one outcome at the $p<0.05$ level). We fit three sets of multiple linear regression models to examine the association of napping intention, frequency, and duration (predictors) with cognitive tests performance and self-rated memory (outcomes). Model 1 adjusted for demographics (age, race, gender, educational attainment), Model 2 
adjusted for Model 1 covariates plus physical health characteristics (BMI category, number of medical conditions), mental health characteristics (PHQ-2, GAD-2), nighttime sleep duration, and sleep medication use, and Model 3 included all Model 2 covariates but excluded persons with a dementia or $\mathrm{AD}$ diagnosis and/or a proxy respondent. In addition, in fully-adjusted models with napping frequency or duration as the primary predictors, we further adjusted for daytime sleepiness (DS) (i.e., Model 2+DS; Model 3+DS). In Model 3, the number of medical conditions variable excluded dementia or AD diagnosis. We made pairwise comparisons between unintentional vs. intentional nappers (reference), frequent vs. infrequent nappers (reference), and long vs. moderate-duration nappers (reference), using linear combination post-estimation analyses. Among participants who reported napping more than rarely, we also explored whether napping characteristics were effect modifiers by fitting models that included the following interaction terms: napping intention*napping frequency, napping intention*nap duration, and nap duration*nap frequency. We performed stratified analyses for any interaction terms with a Wald test $p$-value $<0.10$. We selected an alpha level $<0.05$ for statistical significance in all other analyses. We applied survey weights, which accounted for stratification and clustering resulting from the study design. Analyses were performed using Stata version 15.0 (StataCorp, College Station, TX).

\section{Results}

Participant characteristics by napping type are described in Table 1. Overall, $48.3 \%$ of participants were non-nappers, $23.3 \%$ took intentional naps only, and $28.4 \%$ took unintentional naps. Napping type was significantly associated with age, gender, race, education, BMI, PHQ-2, GAD-2, and number of medical conditions (all $p$ 's<0.05).

Among nappers, $54.5 \%$ were infrequent nappers, and $45.5 \%$ were frequent nappers. Additionally, $45.0 \%$ took short naps ( $\$ 30$ minute), $34.9 \%$ took moderate-duration naps (3160 minutes), and $20.1 \%$ took long naps (>60 minutes). Among nappers, napping intention was associated with napping frequency $(p<0.01)$ and average nap duration $(p<0.05)$. Napping type was associated with nighttime sleep duration and DS (both $p<0.01$ ), but not sleep medication use $(p=0.27)$.

On average, participants recalled $5.1 \pm 0.03$ (standard error) words on the IWR test and 3.8 \pm 0.05 words on the DWR test. The mean CDT and self-rated memory scores were $3.8 \pm 0.03$ and $2.7 \pm 0.02$, respectively.

\section{Napping Characteristics and Cognition}

Intention.-In Model 1 and Model 2, unintentional nappers had lower scores on the IWR test than non-nappers and intentional nappers (Table 2). After excluding participants with dementia and/or proxies (Model 3), unintentional nappers still had lower IWR scores than non-nappers $(\mathrm{B}=-0.19,95 \%$ confidence interval $(\mathrm{CI}):-0.33,-0.05)$ and intentional nappers $(\mathrm{B}=-0.20,95 \% \mathrm{CI}:-0.35,-0.05)$. There was no difference in DWR test performance between non-nappers and unintentional nappers; however, in all models, unintentional nappers had poorer DWR test scores than intentional nappers (Model $3 \mathrm{~B}=-0.23,95 \% \mathrm{CI}$ : $-0.45,-0.01)$. In Model 1 , intentional nappers had poorer self-rated memory than nonnappers ( $\mathrm{B}=0.13,95 \% \mathrm{CI}: 0.02,0.25)$, but not in Models 2 or 3 . Across models, 
unintentional nappers had poorer self-rated memory than non-nappers (Model $3 \mathrm{~B}=0.12$, 95\% CI 0.01,0.23). There were no associations between napping intention and CDT performance.

Frequency.-Frequent nappers had poorer performance on IWR than non-nappers in Model 1 (B=-0.23, 95\% CI: -0.40,-0.05) (Table 3), but not in Models 2+DS or 3+DS. Across models, frequent nappers had poorer self-rated memory than non-nappers (Model 3 $\mathrm{B}=0.15,95 \% \mathrm{CI} 0.03,0.27)$. Frequent nappers had poorer self-rated memory than infrequent nappers in Model 1 ( $\mathrm{B}=0.14,95 \%$ CI $0.03,0.25)$ and $3+\mathrm{DS}(\mathrm{B}=0.13,95 \% \mathrm{CI} 0.01,0.24)$, but not Model 2+DS. Napping frequency was not associated with DWR or CDT performance.

Duration.-In Model 1 and Model 2+DS, long nappers had lower scores on the IWR test than short- (Model 2+DS B=-0.26, 95\% CI: $-0.49,-0.03$ ) and moderate-duration nappers (Model 2+DS B=-0.27, 95\% CI: $-0.51,-0.02$; no association in Model 3+DS) (Table 4). In Model 1, long nappers had poorer DWR test scores than short nappers ( $\mathrm{B}=-0.27,95 \% \mathrm{CI}$ : $-0.53,-0.01$ ), but not in Models 2+DS or 3+DS. In Model 3+DS, compared to short nappers, moderate-duration nappers had better DWR test scores ( $\mathrm{B}=0.24,95 \% \mathrm{CI} 0.01,0.47)$. Long nappers had lower DWR test scores than moderate-duration nappers across models (Model 3 $\mathrm{B}=-0.46,95 \% \mathrm{CI}-0.73,-0.18$ ). In Models 1 and 2+DS, long nappers had lower CDT scores than short nappers (Model 2+DS B=-0.17, 95\% CI: $-0.34,-0.004$ ). Compared to short nappers, long nappers had poorer self-rated memory in Model $1(\mathrm{~B}=0.14,95 \% \mathrm{CI}$ 0.002,0.28), but not in Model 2+DS or 3+DS. CDT scores were not associated with nap duration in Model 3.

Interactions of Napping Characteristics.-When IWR test was the outcome, there were trend-level and significant napping intention*duration interaction terms in Models $2+\mathrm{DS}(p=0.098)$ and $3+\mathrm{DS}(p<0.05)$. After stratifying by napping intention, long nap duration was associated with poorer performance on IWR among intentional nappers, but not unintentional nappers (Table 5). There were no other significant or near-significant interactions between napping characteristics and cognitive outcomes.

\section{Discussion}

We investigated the association of napping characteristics with cognitive variables in a nationally representative sample of community-dwelling U.S. Medicare beneficiaries. After adjustment for potential confounders, compared to non-nappers, unintentional nappers had poorer performance on a test of immediate verbal memory and worse self-rated memory. Further, compared to intentional nappers, unintentional nappers had poorer scores on tests of IWR and DWR. In fully adjusted models, there was no difference in cognitive performance between intentional nappers and non-nappers. However, frequent nappers had poorer selfrated memory than non-nappers. Among nappers, long nap duration was associated with poorer IWR performance when those with a dementia diagnosis and/or proxies were included in the sample. After excluding participants with a dementia diagnosis and/or proxies, some results changed. For instance, long naps were no longer associated with lower CDT scores after excluding participants with dementia or proxies. Additionally, moderateduration naps were associated with better DWR test scores compared to non-nappers, but 
only after participants with dementia or proxies were excluded. Overall, our findings suggest that, among older adults, unintentional, frequent, and longer naps are associated with poorer verbal memory performance or poorer self-rated memory, and that these associations are independent of demographic characteristics, mental and physical health status, and relevant sleep characteristics. The attenuation and loss of some findings after excluding participants with dementia and/or proxies suggest that some of the association between napping and cognition in community-dwelling older adults is due to brain changes associated with dementia. However, most findings remained significant after excluding these participants. Thus, napping may be a marker of risk for preclinical neurodegeneration or of neurodegeneration itself, and perhaps even a contributor to cognitive decline.

By examining the relationship of napping intentionality with cognitive function, our study extends prior research that has largely focused on nap duration or frequency. Our findings are consistent with results indicating that older adults who took unintentional naps had greater cognitive deficits than non-nappers before accounting for other factors including sleep-related characteristics. ${ }^{6}$ In our study, moderate-duration naps were linked to better DWR scores, but only among those who had not reported being diagnosed with dementia and did not have proxies. These findings partially support lab-based studies that have found napping to have positive benefits on cognitive function in healthy older adults. $9,10,21$ However, our findings primarily link naps to poorer cognitive performance. Importantly, in laboratory settings, nap duration can be controlled, and lab-based studies had small samples of healthy older adults, rather than community-based samples with greater medical morbidity. To a degree, our results are also in line with studies linking moderate-duration naps (30-90 minutes) with better cognition ${ }^{13}$ and improvements in cognition. ${ }^{14}$ However, those studies tied moderate-duration naps to better performance on a combined score from three cognitive assessments. Our study diverges from findings linking napping to decreased risk for cognitive decline. ${ }^{8}$ However, conflicting findings may be due to measurement differences. For instance, a U.K.-based study found napping to be protective with respect to cognitive decline, but only included older adults who were cognitively unimpaired at baseline and did not examine napping intention or frequency; ${ }^{8}$ other studies in China only assessed afternoon (i.e., post-lunch) nap duration. ${ }^{13,14}$

When we explored interactions among napping characteristics, we found that, among intentional nappers, those who took long naps had lower IWR scores than short nappers, but there was no such association among unintentional nappers. Given our finding that unintentional napping is associated with poorer IWR compared to intentional napping, this suggests that unintentional naps are linked to poorer cognition regardless of duration, and that long intentional naps may be a marker of, or contributor to, poorer cognition among older adults.

There are multiple mechanisms that may explain the associations observed between napping and cognitive function. Napping is associated with greater risk for several medical conditions including type-II diabetes, ${ }^{22,23}$ incident cardiovascular disease,${ }^{24}$ and greater odds of obesity, ${ }^{25}$ which are themselves associated with cognitive deficits. ${ }^{26-29}$ However, we adjusted for medical morbidity and still found an association between napping and 
cognition. It is possible that napping is a marker of risk for these diseases or preclinical disease, but further research in this domain is needed.

Napping, especially unintentional napping, has also been characterized as a marker of EDS, 15 raising the possibility that EDS drives the association between napping and cognitive performance. Numerous studies have linked EDS to cognitive decline, ${ }^{30-32}$ and those with EDS are more likely to be regular nappers. ${ }^{5}$ Sleep-disordered breathing (SDB) is also prevalent among older people, ${ }^{33}$ and commonly causes DS. Although significant associations between napping characteristics and cognitive performance remained after adjusting for age, gender, self-reported BMI, and DS (risk factors for SDB), we cannot rule out the possibility that SDB is driving our results. This is a limitation in light of evidence supporting SDB as a potential cause of mild cognitive impairment and dementia. ${ }^{34}$

Alternatively, napping may be a marker of neurodegenerative disease. The onset of amyloidbeta deposition was associated with changes in sleep/wake patterns in an AD transgenic mouse model, ${ }^{35}$ and amyloid-beta deposition has been linked to poorer sleep quality and shorter sleep duration in humans. ${ }^{36}$ Moreover, reports of frequent napping have been tied to greater beta-amyloid burden measured in cerebrospinal fluid. ${ }^{37}$ Sleep disturbances are believed to have a reciprocal link with the development of AD pathology, with sleep loss leading to amyloid-beta deposition and vice versa. ${ }^{38}$ Although it is unclear how napping may increase brain amyloid-beta burden, it is feasible that preclinical AD increases napping, and accumulating evidence suggests SDB may promote amyloid deposition in humans. ${ }^{39,40}$

This study had multiple strengths. NHATS is a large, nationally representative study of Medicare beneficiaries in the U.S., and contains measures that permitted analyses of various dimensions of napping behavior in relation to multiple cognitive functioning measures. The large sample also permitted adjustment for important confounders. However, this study had important limitations. We relied on individual items of self-report nap measures which may differ from results from objective measures of sleep (e.g., actigraphy). ${ }^{41}$ In addition, our findings did not take into account the timing of naps, which may interact with other napping characteristics with regard to cognitive performance. Further, our self-report measures of napping and sleepiness consisted of individual items that have not been validated. Similarly, our measure of self-rated memory also relied on self-report, and persons with cognitive impairments may inaccurately rate their memory and napping behaviors. On the other hand, we have no reason to suspect that there would be a systematic bias in our measures (e.g., that those with cognitive impairment would consistently under- or over-estimate their napping frequency). Thus, resulting measurement error would likely bias our results toward the null, rendering them an underestimate of true effects. Confounding by medications other than sleep aids (e.g., alpha- and beta-adrenergic blocking agents, antidepressants) may have affected our findings, and our measure of medical comorbidities may not have captured all conditions that are linked to both cognitive function and napping. Another limitation of this study is our lack of adjustment for multiple comparisons of napping characteristics with cognitive function (i.e., a higher probability of false positives). Subsequent studies are needed to confirm our findings. Finally, because this study is cross-sectional, we cannot draw inferences about a causal direction. 
In summary, we found that napping intention, frequency, and duration are associated with performance on tests of verbal memory, executive function, or self-rated memory, independently of several potential confounders. Although statistically significant, the magnitude of the associations between each of our predictors and outcomes was small. However, our findings highlight napping as a potential marker of risk for cognitive decline, or a contributor to poor cognitive outcomes. Prospective studies of napping are needed that use both self-report and objective sleep measures, such as actigraphy, to quantify napping frequency and duration and relate them to cognitive decline and AD biomarkers. Results from such studies could be leveraged to inform prevention efforts or develop prognostic tools that use sleep measures to anticipate cognitive trajectories.

\section{Acknowledgements:}

The National Health and Aging Trends Study (NHATS) is sponsored by the National Institute on Aging (grant number U01AG032947) through a cooperative agreement with the Johns Hopkins Bloomberg School of Public Health. This work was also supported in part by the National Institute on Aging (grant numbers R01AG050507, R01AG050507-02S1, and T32-AG027668) and the National Institute of Mental Health (T32MH014592-39). Adam Spira agreed to serve as a consultant to Awarables, Inc. in support of an NIH grant.

\section{References}

1. Colby SL, Ortman JM. Projections of the size and composition of the US population: 2014 to 2060. Current Population Reports. 2015(P25-1143). doi: 10.1111/jsr.12313

2. Hebert LE, Weuve J, Scherr PA, Evans DA. Alzheimer disease in the United States (2010-2050) estimated using the 2010 census. Neurology. 2013;80(19):1778-1783. doi: 10.1212/WNL. 0b013e31828726f5 [PubMed: 23390181]

3. Prince M, Bryce R, Albanese E, Wimo A, Ribeiro W, Ferri CP. The global prevalence of dementia: A systematic review and metaanalysis. Alzheimer's \& Dementia. 2013;9(1):63-75.e62. doi: 10.1016/j.jalz.2012.11.007

4. Spira AP, Chen-Edinboro LP, Wu MN, Yaffe K. Impact of sleep on the risk of cognitive decline and dementia. Curr Opin Psychiatry. 2014;27(6):478-483. doi: 10.1097/YCO.0000000000000106 [PubMed: 25188896]

5. Foley D, Vitiello M, Bliwise D, Ancoli-Israel S, Monjan A, Walsh J. Frequent napping is associated with excessive daytime sleepiness, depression, pain, and nocturia in older adults: findings from the National Sleep Foundation '2003 Sleep in America' Poll. Am J Geriatr Psychiatry. 2007;15(4):344350. doi: 10.1097/01.JGP.0000249385.50101.67 [PubMed: 17384317]

6. Ohayon M, Vecchierini M. Daytime sleepiness and cognitive impairment in the elderly population. Arch Intern Med. 2002;162(2):201-208. doi: 10.1001/archinte.162.2.201 [PubMed: 11802754]

7. Ohayon MM, Zulley J. Prevalence of naps in the general population. Sleep Hypn. 1999;1(2):88-97.

8. Keage HAD, Banks S, Yang KL, Morgan K, Brayne C, Matthews FE. What sleep characteristics predict cognitive decline in the elderly? Sleep Med. 2012;13(7):886-892. doi: 10.1016/j.sleep. 2012.02.003 [PubMed: 22560827]

9. Tamaki M, Shirota A, Tanaka H, Hayashi M, Hori T. Effects of a daytime nap in the aged. Psychiatry and clinical neurosciences. 1999;53(2):273-275. doi: 10.1046/j.1440-1819.1999.00548.x [PubMed: 10459710]

10. Campbell SS, Murphy PJ, Stauble TN. Effects of a nap on nighttime sleep and waking function in older subjects. J Am Geriatr Soc. 2005;53(1):48-53. doi: 10.1111/j.1532-5415.2005.53009.x [PubMed: 15667375]

11. Blackwell T, Yaffe K, Ancoli-Israel S, et al. Poor sleep Is associated with impaired cognitive function in older women: The Study of Osteoporotic Fractures. The Journals of Gerontology: Series A. 2006;61(4):405-410. doi: 10.1093/gerona/61.4.405 
12. Cross N, Terpening Z, Rogers NL, et al. Napping in older people 'at risk' of dementia: relationships with depression, cognition, medical burden and sleep quality. J Sleep Res. 2015;24(5):494-502. doi: 10.1111/jsr.12313 [PubMed: 26096839]

13. Li J, Cacchione PZ, Hodgson N, et al. Afternoon napping and cognition in Chinese older adults: findings from the China Health and Retirement Longitudinal Study baseline assessment. J Am Geriatr Soc. 2017;65(2):373-380. doi: 10.1111/jgs.14368 [PubMed: 27995615]

14. Li J, Chang YP, Riegel B, et al. Intermediate, But Not Extended, Afternoon Naps May Preserve Cognition in Chinese Older Adults. J Gerontol A Biol Sci Med Sci. 2017. doi: 10.1093/gerona/ glx069

15. Martin JL, Ancoli-Israel S. Napping in Older Adults. Sleep Med Clin. 2006;1(2):177-186.

16. Kasper J, Freedman V. National Health and Aging Trends Study User Guide: Rounds 1, 2, $3 \& 4$ Final Release. In. Baltimore: Johns Hopkins University School of Public Health; 2015.

17. Ofstedal MB, Fisher GG, Herzog AR. Documentation of cognitive functioning measures in the Health and Retirement Study. Ann Arbor, MI: University of Michigan 2005;10.

18. Schretlen D, Testa S, Pealson G. Clock-drawing test scoring approach from the Calibrated Neuropsychological Normative System. In. Lutz, FL: Psychological Assessment Resources; 2010.

19. World Health Organization. Global database on Body Mass Index: BMI Classification. 2006. In: 2015.

20. Löwe B, Wahl I, Rose M, et al. A 4-item measure of depression and anxiety: validation and standardization of the Patient Health Questionnaire-4 (PHQ-4) in the general population. J Affect Disord. 2010;122(1-2):86-95. doi: 10.1016/j.jad.2009.06.019 [PubMed: 19616305]

21. Tamaki M, Shirota A, Hayashi M, Hori T. Restorative effects of a short afternoon nap ( $<30 \mathrm{~min})$ in the elderly on subjective mood, performance and eeg activity. Sleep research online : SRO. 2000;3(3):131-139. [PubMed: 11382911]

22. Lam K-bH, Jiang C, Thomas G, et al. Napping is associated with increased risk of type 2 diabetes: the Guangzhou Biobank Cohort Study. Sleep. 2010;33(3):402-407. [PubMed: 20337199]

23. Leng Y, Cappuccio FP, Surtees PG, Luben R, Brayne C, Khaw KT. Daytime napping, sleep duration and increased 8-year risk of type 2 diabetes in a British population. Nutr Metab Cardiovasc Dis. 2016;26(11):996-1003. doi: 10.1016/j.numecd.2016.06.006 [PubMed: 27484757]

24. Yang L, Yang H, He M, et al. Longer sleep duration and midday napping are associated with a higher risk of CHD incidence in middle-aged and older Chinese: the Dongfeng-Tongji Cohort Study. Sleep. 2016;39(3):645-652. doi: 10.5665/sleep.5544 [PubMed: 26564127]

25. Patel SR, Hayes AL, Blackwell T, et al. The association between sleep patterns and obesity in older adults. Int J Obes (Lond). 2014;38(9):1159-1164. doi: 10.1038/ijo.2014.13 [PubMed: 24458262]

26. Wennberg AM, Gottesman RF, Kaufmann CN, et al. Diabetes and cognitive outcomes in a nationally representative sample: the National Health and Aging Trends Study. Int Psychogeriatr. 2014;26(10):1729-1735. doi: 10.1017/S1041610214001380 [PubMed: 25075535]

27. Okereke OI, Kang JH, Cook NR, et al. Type 2 diabetes mellitus and cognitive decline in two large cohorts of community-dwelling older adults. J Am Geriatr Soc. 2008;56(6):1028-1036. doi: 10.1111/j.1532-5415.2008.01686.x [PubMed: 18384580]

28. Zeki Al Hazzouri A, Haan MN, Neuhaus JM, et al. Cardiovascular risk score, cognitive decline, and dementia in older Mexican Americans: the role of sex and education. Journal of the American Heart Association. 2013;2(2):e004978. doi: 10.1161/jaha.113.004978 [PubMed: 23608609]

29. Hassing LB, Dahl AK, Pedersen NL, Johansson B. Overweight in midlife is related to lower cognitive function 30 years later: a prospective study with longitudinal assessments. Dementia and geriatric cognitive disorders. 2010;29(6):543-552. [PubMed: 20606436]

30. Foley D, Monjan A, Masaki K, et al. Daytime sleepiness is associated with 3-Year incident dementia and cognitive decline in older Japanese-American men. J Am Geriatr Soc. 2001;49(12): 1628-1632. doi: 10.1111/j.1532-5415.2001.49271.x [PubMed: 11843995]

31. Jaussent I, Bouyer J, Ancelin ML, et al. Excessive sleepiness is predictive of cognitive decline in the elderly. Sleep. 2012;35(9):1201-1207. doi: 10.5665/sleep.2070 [PubMed: 22942498]

32. Merlino G, Piani A, Gigli GL, et al. Daytime sleepiness is associated with dementia and cognitive decline in older Italian adults: a population-based study. Sleep Med. 2010;11(4):372-377. doi: 10.1016/j.sleep.2009.07.018 [PubMed: 20219426] 
33. McMillan A, Morrell MJ. Sleep disordered breathing at the extremes of age: the elderly. Breathe. 2016;12(1):50-60. doi: 10.1183/20734735.003216 [PubMed: 27064674]

34. Yaffe K, Laffan AM, Harrison SL, et al. Sleep-disordered breathing, hypoxia, and risk of mild cognitive impairment and dementia in older women. JAMA. 2011;306(6):613-619. doi: 10.1001/ jama.2011.1115 [PubMed: 21828324]

35. Roh JH, Huang Y, Bero AW, et al. Disruption of the sleep-wake cycle and diurnal fluctuation of beta-amyloid in mice with Alzheimer's disease pathology. Sci Transl Med. 2012;4(150):150ra122. doi: 10.1126/scitranslmed.3004291

36. Spira AP, Gamaldo AA, An Y, et al. Self-Reported Sleep and $\beta$-Amyloid Deposition in Community-Dwelling Older Adults. JAMA neurology. 2013;70(12):1537-1543. [PubMed: 24145859]

37. Ju Y-ES, McLeland JS, Toedebusch CD, et al. Sleep quality and preclinical Alzheimer Disease. JAMA Neurol. 2013;70(5):587-593. doi: 10.1001/jamaneurol.2013.4258 [PubMed: 23479184]

38. Ju Y-ES, Lucey BP, Holtzman DM. Sleep and Alzheimer disease pathology-a bidirectional relationship. Nat Rev Neurol. 2014;10(2):115-119. doi: 10.1038/nrneurol.2013.269 [PubMed: 24366271]

39. Osorio RS, Ayappa I, Mantua J, et al. Interaction between sleep-disordered breathing and apolipoprotein E genotype on cerebrospinal fluid biomarkers for Alzheimer's disease in cognitively normal elderly individuals. Neurobiol Aging. 2014;35(6):1318-1324. doi: 10.1016/ j.neurobiolaging.2013.12.030 [PubMed: 24439479]

40. Spira AP, Yager C, Brandt J, et al. Objectively measured sleep and beta-amyloid burden in older adults: A pilot study. SAGE Open Med. 2014;2. doi: 10.1177/2050312114546520

41. Dautovich ND, McCrae CS, Rowe M. Subjective and objective napping and sleep in older adults: are evening naps "bad" for nighttime sleep? J Am Geriatr Soc. 2008;56(9):1681-1686. doi: 10.1111/j.1532-5415.2008.01822.x [PubMed: 18691289] 


\section{Key Points}

- Among community-dwelling older adults in the United States, unintentional, frequent, and longer naps were independently associated with poorer performance on cognitive tests or poorer self-rated memory.

- In this population, napping may have utility in the clinical setting as a marker for those who are at greater risk for neurodegeneration.

- This research on napping in older adults is important, given the need to identify those at higher risk for dementia, facilitate care planning, and potentially prevent or slow progression of cognitive decline.

- $\quad$ Although most associations between napping and cognition remained after the exclusion of those with dementia and/or proxy respondents, some findings attenuated or became non-significant. Additionally, moderate-duration naps were associated with better performance on a test of verbal memory, but only among those without a dementia diagnosis and/or a proxy respondent. 
Table 1.

Participant characteristics (Weighted column $\%$ or mean \pm standard error)

\begin{tabular}{|c|c|c|c|c|c|}
\hline Unweighted $\mathbf{N}$ & Total Sample & $\begin{array}{c}\text { Non- } \\
\text { nappers }\end{array}$ & $\begin{array}{c}\text { Intentional } \\
\text { Napping }\end{array}$ & $\begin{array}{l}\text { Unintentional } \\
\text { Napping }\end{array}$ & P-value \\
\hline & $100 \%$ & $48.3 \%$ & $23.3 \%$ & $28.4 \%$ & \\
\hline & $(\mathrm{N}=2,549)$ & $(\mathrm{n}=1,161)$ & $(\mathrm{n}=562)$ & $(\mathrm{n}=826)$ & \\
\hline \multicolumn{6}{|l|}{ Age } \\
\hline $65-69$ & 12.3 & 12.7 & 13.4 & 11.1 & \multirow[t]{6}{*}{$<0.001$} \\
\hline $70-74$ & 33.2 & 36.5 & 34.1 & 26.9 & \\
\hline $75-59$ & 24.1 & 24.9 & 21.7 & 24.6 & \\
\hline $80-84$ & 16.9 & 15.1 & 16.4 & 20.1 & \\
\hline $85-89$ & 9.3 & 7.8 & 10.0 & 11.2 & \\
\hline $90+$ & 4.2 & 3.0 & 4.4 & 6.1 & \\
\hline \multicolumn{6}{|l|}{ Gender } \\
\hline Male & 42.9 & 35.5 & 49.1 & 50.5 & \multirow[t]{2}{*}{$<0.001$} \\
\hline Female & 57.1 & 64.5 & 50.9 & 49.5 & \\
\hline \multicolumn{6}{|l|}{ Race } \\
\hline White, Non-Hispanic & 82.0 & 83.5 & 88.0 & 74.5 & \multirow[t]{4}{*}{$<0.001$} \\
\hline Black, Non-Hispanic & 7.8 & 6.9 & 4.0 & 12.4 & \\
\hline Hispanic & 6.3 & 6.3 & 4.9 & 7.2 & \\
\hline Other & 3.9 & 3.3 & 3.1 & 5.9 & \\
\hline \multicolumn{6}{|l|}{ Education Level } \\
\hline$<$ High School & 18.8 & 17.3 & 14.8 & 24.6 & \multirow[t]{3}{*}{$<0.001$} \\
\hline High School Graduate & 25.9 & 25.9 & 21.5 & 29.5 & \\
\hline >High School & 55.3 & 56.8 & 63.7 & 45.9 & \\
\hline \multicolumn{6}{|l|}{ BMI (in $\mathrm{kg} / \mathrm{m}^{2}$ ) } \\
\hline$<18.5$ & 1.8 & 2.0 & 1.8 & 1.5 & \multirow[t]{4}{*}{$<0.001$} \\
\hline $18.5-24.9$ & 30.7 & 34.2 & 28.2 & 26.9 & \\
\hline $25-29.9$ & 38.5 & 41.2 & 35.8 & 36.0 & \\
\hline 230.0 & 29.0 & 22.6 & 34.2 & 35.6 & \\
\hline PHQ-2 & $0.84 \pm 0.03$ & $0.69 \pm 0.04$ & $0.85 \pm 0.05$ & $1.07 \pm 0.05$ & $<0.001$ \\
\hline GAD-2 & $0.79 \pm 0.03$ & $0.74 \pm 0.04$ & $0.77 \pm 0.05$ & $0.90 \pm 0.05$ & $<0.05$ \\
\hline \multicolumn{6}{|l|}{ No. of Medical Conditions } \\
\hline 0 or 1 & 27.4 & 31.3 & 27.8 & 20.2 & \multirow[t]{4}{*}{$<0.001$} \\
\hline 2 & 28.6 & 29.3 & 30.4 & 26.1 & \\
\hline 3 & 22.5 & 21.4 & 21.0 & 25.5 & \\
\hline 4 or more & 21.5 & 18.0 & 20.8 & 28.2 & \\
\hline \multicolumn{6}{|l|}{ Nap Frequency ${ }^{a}$} \\
\hline Infrequent & 54.5 & -- & 50.0 & 58.2 & \multirow[t]{2}{*}{$<0.01$} \\
\hline Frequent & 45.5 & -- & 50.0 & 41.8 & \\
\hline
\end{tabular}




\begin{tabular}{|c|c|c|c|c|c|}
\hline Unweighted $\mathbf{N}$ & Total Sample & $\begin{array}{c}\text { Non- } \\
\text { nappers }\end{array}$ & $\begin{array}{l}\text { Intentional } \\
\text { Napping }\end{array}$ & $\begin{array}{l}\text { Unintentional } \\
\text { Napping }\end{array}$ & P-value \\
\hline Average Nap Duration ${ }^{a, b}$ & & -- & & & \\
\hline Short & 45.0 & -- & 40.5 & 48.8 & \multirow[t]{3}{*}{$<0.05$} \\
\hline Moderate & 34.9 & -- & 38.6 & 31.8 & \\
\hline Long & 20.1 & -- & 20.9 & 19.4 & \\
\hline Nighttime Sleep Duration & $6.99 \pm 0.04$ & $6.98 \pm 0.05$ & $7.19 \pm 0.07$ & $6.86 \pm 0.06$ & $<0.01$ \\
\hline \multicolumn{6}{|l|}{ Daytime Sleepiness } \\
\hline Never & 34.3 & 44.7 & 31.7 & 18.4 & \multirow[t]{5}{*}{$<0.001$} \\
\hline Rarely & 30.8 & 36.3 & 32.5 & 19.9 & \\
\hline Some Days & 24.6 & 14.0 & 26.0 & 41.6 & \\
\hline Most Days & 6.4 & 2.9 & 7.5 & 11.6 & \\
\hline Every Day & 3.9 & 2.1 & 2.3 & 8.5 & \\
\hline \multicolumn{6}{|l|}{ Sleep Medication Use } \\
\hline Never & 69.8 & 70.1 & 68.4 & 70.5 & \multirow[t]{5}{*}{0.27} \\
\hline Rarely & 8.0 & 8.7 & 8.1 & 6.7 & \\
\hline Some Nights & 6.3 & 5.8 & 7.2 & 6.5 & \\
\hline Most Nights & 3.3 & 2.9 & 5.0 & 2.6 & \\
\hline Every Night & 12.6 & 12.5 & 11.3 & 13.7 & \\
\hline
\end{tabular}

Notes. Patient Health Questionnnaire-2 (Range: 0-6). Generalized Anxiety Disorder Scale-2 (Range: 0-6).

a Excluded non-nappers.

$b$

${ }^{b}$ Average nap duration by category were the following: short nappers: $\leq 30$ minutes, moderate-duration nappers: $31-60$ minutes, long nappers: $>60$ minutes. 
Table 2.

Adjusted associations between napping intention and cognition

\begin{tabular}{|c|c|c|c|c|}
\hline Napping Type & $\begin{array}{l}\text { Immediate Word } \\
\text { Recall }\end{array}$ & Delayed Word Recall & Clock Drawing Test & $\begin{array}{c}\text { Poorer Self-Rated } \\
\text { Memory }\end{array}$ \\
\hline & B $(95 \% \mathrm{CI})$ & B $(95 \%$ CI $)$ & B $(95 \%$ CI $)$ & B $(95 \% \mathrm{CI})$ \\
\hline & \multicolumn{4}{|c|}{ MODEL $1^{a}$} \\
\hline & $\mathrm{n}=2,523$ & $\mathrm{n}=2,518$ & $\mathrm{n}=2,486$ & $\mathrm{n}=2,493$ \\
\hline Non-nappers & Ref & Ref & Ref & Ref \\
\hline Intentional & $-0.02(-0.19,0.15)$ & $0.10(-0.11,0.31)$ & $0.03(-0.07,0.14)$ & $0.13(0.02,0.25)^{*}$ \\
\hline Unintentional & $-0.26(-0.41,-0.10)^{* *}$ & $-0.17(-0.35,-0.00002)$ & $0.02(-0.08,0.11)$ & $0.19(0.09,0.28)^{* *}$ \\
\hline Intentional & Ref & Ref & Ref & Ref \\
\hline \multirow[t]{3}{*}{ Unintentional } & $-0.24(-0.42,-0.05)^{*}$ & $-0.28(-0.50,-0.05)^{*}$ & $-0.02(-0.14,0.10)$ & $0.06(-0.06,0.17)$ \\
\hline & \multicolumn{4}{|c|}{$\operatorname{MODEL} 2^{b}$} \\
\hline & $\mathrm{n}=2,329$ & $\mathrm{n}=2,325$ & $\mathrm{n}=2,307$ & $\mathrm{n}=2,310$ \\
\hline Non-nappers & Ref & Ref & Ref & Ref \\
\hline Intentional & $0.03(-0.13,0.20)$ & $0.16(-0.06,0.38)$ & $0.05(-0.06,0.16)$ & $0.11(-0.01,0.24)$ \\
\hline Unintentional & $-0.23(-0.38,-0.08)^{* * *}$ & $-0.14(-0.32,0.04)$ & $0.03(-0.06,0.12)$ & $0.13(0.02,0.24)^{*}$ \\
\hline Intentional & Ref & Ref & Ref & Ref \\
\hline \multirow[t]{3}{*}{ Unintentional } & $-0.26(-0.43,-0.10)^{* *}$ & $-0.30(-0.53,-0.07)^{*}$ & $-0.02(-0.14,0.11)$ & $0.02(-0.10,0.13)$ \\
\hline & \multicolumn{4}{|c|}{ MODEL $3^{c}$} \\
\hline & $\mathrm{n}=2,225$ & $\mathrm{n}=2,225$ & $\mathrm{n}=2,206$ & $\mathrm{n}=2,239$ \\
\hline Non-nappers & Ref & Ref & Ref & Ref \\
\hline Intentional & $0.01(-0.15,0.17)$ & $0.13(-0.08,0.35)$ & $0.06(-0.05,0.17)$ & $0.11(-0.01,0.24)$ \\
\hline Unintentional & $-0.19(-0.33,-0.05)^{* *}$ & $-0.10(-0.26,0.07)$ & $0.06(-0.03,0.15)$ & $0.12(0.01,0.23)^{*}$ \\
\hline Intentional & Ref & Ref & Ref & Ref \\
\hline Unintentional & $-0.20(-0.35,-0.05)^{* *}$ & $-0.23(-0.45,-0.01)^{*}$ & $0.001(-0.12,0.12)$ & $0.01(-0.11,0.12)$ \\
\hline
\end{tabular}

Notes.

${ }^{a}$ Model 1 adjusted for age, race, gender, and education.

${ }^{b}$ Model 2 adjusted for Model 1 covariates + number of medical conditions, BMI category, GAD-2, PHQ-2, nighttime sleep duration, and use of medication for sleep.

${ }^{c}$ Model 3 adjusted for Model 2 covariates and excludes those with dementia and proxy respondents

*

p-value $<0.05$;

**

p-value $<0.01$ 
Table 3.

Adjusted associations between napping frequency and cognition

\begin{tabular}{|c|c|c|c|c|}
\hline Napping Frequency & $\begin{array}{l}\text { Immediate Word } \\
\text { Recall }\end{array}$ & Delayed Word Recall & Clock Drawing Test & $\begin{array}{c}\text { Poorer Self-Rated } \\
\text { Memory }\end{array}$ \\
\hline & $\mathrm{B}(95 \% \mathrm{CI})$ & B $(95 \% \mathrm{CI})$ & $\mathrm{B}(95 \% \mathrm{CI})$ & B $(95 \% \mathrm{CI})$ \\
\hline & \multicolumn{4}{|c|}{ MODEL $1^{a}$} \\
\hline & $\mathrm{n}=2,523$ & $\mathrm{n}=2,518$ & $\mathrm{n}=2,486$ & $\mathrm{n}=2,493$ \\
\hline Non-nappers & Ref & Ref & Ref & Ref \\
\hline Infrequent & $-0.08(-0.24,0.07)$ & $0.03(-0.17,0.23)$ & $0.07(-0.03,0.17)$ & $0.10(-0.01,0.20)$ \\
\hline Frequent & $-0.23(-0.40,-0.05)^{*}$ & $-0.14(-0.34,0.06)$ & $-0.03(-0.13,0.06)$ & $0.24(0.14,0.34)^{* *}$ \\
\hline Infrequent & Ref & Ref & Ref & Ref \\
\hline \multirow[t]{3}{*}{ Frequent } & $-0.14(-0.33,0.05)$ & $-0.17(-0.42,0.09)$ & $-0.11(-0.22,0.005)$ & $0.14(0.03,0.25)^{*}$ \\
\hline & \multicolumn{4}{|c|}{ MODEL $2^{b}+$ Daytime Sleepiness } \\
\hline & $\mathrm{n}=2,327$ & $\mathrm{n}=2,323$ & $\mathrm{n}=2,305$ & $\mathrm{n}=2,308$ \\
\hline Non-nappers & Ref & Ref & Ref & Ref \\
\hline Infrequent & $-0.08(-0.24,0.08)$ & $0.05(-0.18,0.27)$ & $0.06(-0.04,0.15)$ & $0.03(-0.09,0.15)$ \\
\hline Frequent & $-0.15(-0.32,0.03)$ & $-0.03(-0.26,0.20)$ & $-0.02(-0.12,0.09)$ & $0.14(0.02,0.27)^{*}$ \\
\hline Infrequent & Ref & Ref & Ref & Ref \\
\hline \multirow[t]{3}{*}{ Frequent } & $-0.07(-0.25,0.12)$ & $-0.08(-0.33,0.18)$ & $-0.07(-0.19,0.03)$ & $0.12(-0.001,0.23)$ \\
\hline & \multicolumn{4}{|c|}{ MODEL $3^{c}+$ Daytime Sleepiness } \\
\hline & $\mathrm{n}=2,223$ & $\mathrm{n}=2,223$ & $\mathrm{n}=2,204$ & $\mathrm{n}=2,237$ \\
\hline Non-nappers & Ref & Ref & Ref & Ref \\
\hline Infrequent & $-0.09(-0.25,0.07)$ & $0.03(-0.18,0.25)$ & $0.07(-0.02,0.17)$ & $0.02(-0.10,0.14)$ \\
\hline Frequent & $-0.12(-0.31,0.07)$ & $0.0006(-0.23,0.23)$ & $0.002(-0.11,0.11)$ & $0.15(0.03,0.27)^{*}$ \\
\hline Infrequent & Ref & Ref & Ref & Ref \\
\hline Frequent & $-0.03(-0.24,0.18)$ & $-0.03(-0.30,0.24)$ & $-0.07(-0.18,0.04)$ & $0.13(0.01,0.24)^{*}$ \\
\hline
\end{tabular}

Notes.

${ }^{a}$ Model 1 adjusted for age, race, gender, and education.

${ }^{b}$ Model 2 adjusted for Model 1 covariates + number of medical conditions, BMI category, GAD-2, PHQ-2, nighttime sleep duration, and use of medication for sleep.

${ }^{c}$ Model 3 adjusted for Model 2 covariates and excludes those with dementia and proxy respondents

p-value $<0.05$;

p-value $<0.01$ 
Table 4:

Adjusted associations between average nap duration and cognition

\begin{tabular}{|c|c|c|c|c|}
\hline $\begin{array}{l}\text { Nap Duration (by } \\
\text { group) }{ }^{a}\end{array}$ & $\begin{array}{l}\text { Immediate Word } \\
\text { Recall }\end{array}$ & Delayed Word Recall & Clock Drawing Test & $\begin{array}{c}\text { Poorer Self-Rated } \\
\text { Memory }\end{array}$ \\
\hline & B $(95 \% \mathrm{CI})$ & B $(95 \% \mathrm{CI})$ & B $(95 \% \mathrm{CI})$ & B $(95 \% \mathrm{CI})$ \\
\hline & \multicolumn{4}{|c|}{ MODEL $1 b$} \\
\hline & $\mathrm{n}=1,329$ & $\mathrm{n}=1,327$ & $\mathrm{n}=1,308$ & $\mathrm{n}=1,298$ \\
\hline Short & Ref & Ref & Ref & Ref \\
\hline Moderate & $0.0008(-0.21,0.21)$ & $0.21(-0.02,0.44)$ & $-0.11(-0.26,0.03)$ & $0.06(-0.06,0.18)$ \\
\hline Long & $-0.34(-0.59,-0.10)^{* *}$ & $-0.27(-0.53,-0.01)^{*}$ & $-0.18(-0.34,-0.02)^{*}$ & $0.14(0.002,0.28)^{*}$ \\
\hline Moderate & Ref & Ref & Ref & Ref \\
\hline \multirow[t]{3}{*}{ Long } & $-0.34(-0.60,-0.08)^{*}$ & $-0.48(-0.75,-0.21)^{* *}$ & $-0.07(-0.25,0.12)$ & $0.08(-0.07,0.23)$ \\
\hline & \multicolumn{4}{|c|}{ MODEL $2^{c}+$ Daytime Sleepiness } \\
\hline & $\mathrm{n}=1,229$ & $\mathrm{n}=1,227$ & $\mathrm{n}=1,217$ & $\mathrm{n}=1,208$ \\
\hline Short & Ref & Ref & Ref & Ref \\
\hline Moderate & $0.01(-0.18,0.20)$ & $0.23(-0.0006,0.46)$ & $-0.10(-0.25,0.05)$ & $0.05(-0.09,0.19)$ \\
\hline Long & $-0.26(-0.49,-0.03)^{*}$ & $-0.23(-0.52,0.06)$ & $-0.17(-0.34,-0.004)^{*}$ & $0.06(-0.08,0.21)$ \\
\hline Moderate & Ref & Ref & Ref & Ref \\
\hline \multirow[t]{3}{*}{ Long } & $-0.27(-0.51,-0.02)^{*}$ & $-0.46(-0.74,-0.17)^{* *}$ & $-0.07(-0.27,0.13)$ & $0.01(-0.14,0.17)$ \\
\hline & \multicolumn{4}{|c|}{ MODEL $3^{d}+$ Daytime Sleepiness } \\
\hline & $\mathrm{n}=1,158$ & $\mathrm{n}=1,159$ & $\mathrm{n}=1,149$ & $\mathrm{n}=1,166$ \\
\hline Short & Ref & Ref & Ref & Ref \\
\hline Moderate & $0.004(-0.19,0.19)$ & $0.24(0.01,0.47)^{*}$ & $-0.09(-0.24,0.06)$ & $0.05(-0.08,0.19)$ \\
\hline Long & $-0.22(-0.46,0.02)$ & $-0.21(-0.49,0.06)$ & $-0.14(-0.32,0.03)$ & $0.05(-0.10,0.20)$ \\
\hline Moderate & Ref & Ref & Ref & Ref \\
\hline Long & $-0.23(-0.45,-0.00004)$ & $-0.46(-0.73,-0.18)^{* * *}$ & $-0.05(-0.28,0.17)$ & $-0.003(-0.17,0.17)$ \\
\hline
\end{tabular}

Notes. Excluded non-nappers.

${ }^{a}$ Average nap duration by category were the following: short nappers: 30 minutes, moderate-duration nappers: $31-60$ minutes, long nappers: $>60$ minutes.

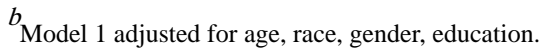

${ }^{c}$ Model 2 adjusted for Model 1 covariates + number of medical conditions, BMI category, GAD-2, PHQ-2, nighttime sleep duration, and use of medication for sleep.

${ }^{d}$ Model 3 adjusted for Model 2 covariates and excludes those with dementia and proxy respondents

*

p-value $<0.05$;

**

p-value $<0.01$ 
Table 5.

Significant Interactions of Napping Intention with Nap Duration: Immediate Word Recall

\begin{tabular}{l|c|c}
\hline & Intentional Nappers & Unintentional Nappers \\
\hline Nap Duration (by group) $^{a}$ & $\mathrm{~B}(95 \% \mathrm{CI})$ & $\mathrm{B}(95 \% \mathrm{CI})$ \\
\hline & \multicolumn{2}{|c}{ Model $2^{b}+$ Daytime Sleepiness } \\
\hline Short & $\mathrm{n}=519$ & $\mathrm{n}=710$ \\
\hline Moderate & $\mathrm{Ref}$ & $\mathrm{Ref}$ \\
\hline Long & $-0.04(-0.34,0.25)$ & $0.0002(-0.23,0.23)$ \\
\hline & $-0.54(-0.85,-0.23)^{* * *}$ & $-0.01(-0.33,0.31)$ \\
\hline & $\mathrm{Model} 3^{c}+$ Daytime Sleepiness \\
\hline Short & $\mathrm{n}=508$ & $\mathrm{n}=650$ \\
\hline Moderate & Ref & Ref \\
\hline Long & $-0.03(-0.34,0.27)$ & $0.007(-0.19,0.21)$ \\
\hline
\end{tabular}

Notes. Excluded non-nappers.

${ }^{a}$ Average nap duration by category were the following: short nappers: $\leq 30$ minutes, moderate-duration nappers: $31-60$ minutes, long nappers: >60 minutes.

${ }^{b}$ Model 2 adjusted for Model 1 covariates + number of medical conditions, BMI category, GAD-2, PHQ-2, nighttime sleep duration, and use of medication for sleep.

${ }^{c}$ Model 3 adjusted for Model 2 covariates and excludes those with dementia and proxy respondents

*

p-value $<0.05$;

***

p-value $<0.01$ 